\title{
Vivência e concepção da mulher acerca do climatério: Uma revisão bibliográfica
}

\author{
Women's experience and conception about the climate: A bibliographic review \\ Experiencia y concepción de mujeres sobre el clima: Uma revisón bibliográfica
}

\author{
Josielen Barroso Leal Maciel \\ ORCID: https://orcid.org/0000-0001-9237-2466 \\ Universidade Estadual do Maranhão, Brasil \\ E-mail: usuariodavid52@gmail.com \\ Alana Jéssyca Costa Sipaúba \\ ORCID: https://orcid.org/0000-0003-0127-118X \\ Faculdade de Ciências e Tecnologias do Maranhão, Brasil \\ E-mail: alanacolinas@ hotmail.com \\ Thátila Larissa da Cruz Andrade \\ ORCID: https://orcid.org/0000-0002-8030-3516 \\ Universidade Estadual do Maranhão, Brasil \\ E-mail: thatilaandrade@gmail.com \\ Helen Lucy Maria Rocha Barroso \\ ORCID: https://orcid.org/0000-0001-8537-7808 \\ Universidade Estadual do Maranhão, Brasil \\ E-mail: helenlucy.h140@gmail.com \\ Jordânia Ferreira de Amorim \\ ORCID: https://orcid.org/0000-0002-1415-6917 \\ Universidade Federal do Piauí, Brasil \\ E-mail: jordania_amorim@hotmail.com \\ Klécia de Sousa Marques da Silva \\ ORCID: https://orcid.org/0000-0001-5331-8934 \\ Universidade Federal do Maranhão, Brasil \\ E-mail: kleciamarques19@gmail.com \\ Alice da Silva Souza \\ ORCID: https://orcid.org/0000-0002-9803-844X \\ Universidade Estadual do Maranhão, Brasil \\ E-mail: alice_12_flor@live.com
}

\begin{abstract}
Resumo
Introdução: $\mathrm{O}$ climatério trata-se de um período em que acontece a transição do período reprodutivo para o não reprodutivo, podendo ocorrer entre 40 e 65 anos. Objetivo: Conhecer a concepção das mulheres a respeito do climatério as eventuais consequências desencadeada na vida sexual, emocional, através de uma revisão bibliográfica. Metodologia: Para o desenvolvimento desta presente pesquisa realizou-se uma revisão bibliográfica, onde foram utilizadas técnicas de coletas de dados através do pensamento e das ideias dos autores. Resultados e Discussão: Foram revisados onze (11) artigos científicos, sete (07) revisões bibliográficas. As mudanças durante o climatério causam um enfrentamento durante este ciclo, além dos fatores hormonais, interferindo no emocional, social e sexual. Conclusão: Foi evidenciado que cada mulher vivencia o climatério de forma singular, tanto como a idade em que se inicia, e a forma que se instala, sendo natural ou cirúrgica.
\end{abstract}

Palavras-chave: Climatério; Menopausa; Saúde da mulher; Saúde sexual.

\begin{abstract}
Introduction: Climacteric is a period in which the transition from the reproductive to the non-reproductive period takes place, and can occur between 40 and 65 years. Objective: To know the conception of women regarding the climacteric period and the possible consequences on sexual and emotional life, through a literature review. Methodology: For the development of this research, a bibliographic review was performed, where techniques of data collection through the authors' thoughts and ideas were used. Results and Discussion: Dose (11) scientific articles, seven (07) bibliographic review. Changes during the climacteric cycle cause a confrontation during this cycle beyond the hormonal factors, interfering in the emotional, social and sexual. Conclusion: It has been shown that each woman experiences the climacteric in a unique way, as much as the age at which it starts, and the way it settles down, being natural or surgical.
\end{abstract}

Keywords: Climacteric; Menopause; Women's health; Sexual health. 


\begin{abstract}
Resumen
Introducción: El climatérico es un período en el cual ocorre la transición del período reproductivo al no reproductivo, pudiendo ocurrir entre los 40 y 65 años. Objetivo: Conocer la conceptión de la mujer sobre el climatério y las posibles consecuencias desatadas en la vida sexual y emocional, através de una revisión bibliográfica, utilizando técnicas de recolección de datos através del pensamento e ideas de los autores. Resultados y Discusión: Se revisaron (11) artículos científicos, se revisaron siete (07) revisiones bibliográficas. Los câmbios durante el climatérico provocan un enfrentamento durante este ciclo además de factores hormonales interfiriendo en lo emocional, social y sexual. Conclusión: Se evidenció que cada mujer vive el climatérico de una manera única, tanto como la edad en la que se inicia y la forma en que se asienta, sea natural o quirúrgica.
\end{abstract}

Palabras clave: Climatérico; Menopausia; La salud de la mujer; Salud sexual.

\title{
1. Introdução
}

O climatério trata-se de um período em que acontece a transição do período reprodutivo para o não reprodutivo, podendo ocorrer entre 40 e 65 anos. Dividido em três fases: fase pré-menopausa, fase menacme e fase senectude ou senescência (Brasil, 2008; Brasil, 2016).

Nesse período, ocorre a menopausa na qual é definida como um intervalo permanente da menstruação e reconhecida após 12 meses sucessivos de amenorreia. Desse modo, é definido pela Organização Mundial de Saúde - OMS como um período fisiológico e não patológico (Febrasgo, 2010; Santos et al., 2007).

Quando esta fase acontece antes dos 40 anos de idade ela é considerada precoce, e tardia após os 55 anos de idade, dependendo da saúde e do estilo de vida da mulher. (Febrasgo, 2010; Ferreira, Silva \& de Almeida, 2015). Podendo ocorrer de uma forma "não natural" por intervenção cirúrgica (Santos et al., 2007).

A irregularidade menstrual é universal e os fogachos e suores noturnos também são bastante frequentes, típicos deste período. É importante frisar, que as queixas que mais influenciam na qualidade de vida da mulher neste período são as de ordem psicossocial e afetiva (Brasil, 2016).

Os sintomas vasomotores, que são representados por fogachos, sudorese e insônia, podem ser verificados em quase 75\% das mulheres perimenopáusicas. Durante os episódios de fogachos nota-se um rápido fluxo sanguíneo e a temperatura da pele poderá aumentar entre $1 \mathrm{a} 7^{\circ} \mathrm{c}$. Tendo duração de 3 a 5 anos e constitui a marca registrada da perimenopausa, podendo ocorrer de forma diária, semanal ou mensal (Petel et al., 2003 apud Oliveira Júnior, 2016).

No Brasil durante o século XX a saúde da mulher foi implantada e seguindo um processo de evolução considerando o início da assistência a este grupo populacional na década de 70. Neste período o Ministério da Saúde - MS aderia restritamente à saúde materna ou à ausência de agravos associados à reprodução biológica (Brasil, 2008).

Em 1999, a Área Técnica de Saúde da Mulher do Ministério da Saúde - MS incorporou no seu planejamento a atenção à saúde da mulher acima de 50 anos. Porém, nenhuma ação específica foi colocada em prática naquela oportunidade. Foi realizado um balanço institucional em 2002, onde exibiu, entre outras, esta lacuna, considerando necessário superá-la (Brasil, 2008).

Em 2003, houve uma necessidade da elaboração de políticas voltadas a ações a saúde da mulher no climatério e incluindo um capítulo sobre este tema no documento Política Nacional Integral a Saúde da Mulher - Princípios e Diretrizes. Tendo como objetivo implantar e programar a atenção à saúde da mulher no climatério, em nível nacional, tendo em vista, ampliar o acesso e qualificar com ações voltadas a saúde da mulher no climatério (Brasil, 2008).

O período da menopausa causa desgastes emocionais, sendo associada à condição de vida, história reprodutiva, carga de serviço, hábitos de alimentação, predisposição a infecção, falta de acesso a serviços de saúde e informações sobre o assunto, levando a mulher a um sofrimento demasiado neste período (Valença \& Germano, 2010).

Entender o que esta mulher enfrenta neste período é de grande relevância, dados atuais tem mostrado que o aumento 
dos sintomas e problemas da mulher neste período reflete circunstancias sociais e pessoais e não apenas acontecimentos endócrinos do climatério. (Unasus, 2013).

Outras variáveis relacionadas à discriminação e aos preconceitos também compõem o processo de saúde-doença e aumentam a vulnerabilidade frente a determinados agravos que estão relacionados com situações de discriminação. (Brasil, 2008). Diante disso a questão norteadora para realização deste trabalho foi, qual a concepção destas mulheres durante o climatério?

\section{Objetivo}

Conhecer a concepção das mulheres a respeito do climatério, as eventuais consequências desencadeadas na vida sexual e emocional, através de uma revisão bibliográfica.

\section{Metodologia}

Realizou-se uma revisão bibliográfica, onde foram utilizadas técnicas de coletas de dados através do pensamento e das ideias dos autores, de acordo com Vianna, 2001 "a revisão bibliográfica é a base que sustenta qualquer pesquisa científica". Tal pesquisa não busca enumerar ou medir eventos, mas ela serve para obter dados descritivos que expressam os sentidos dos fenômenos. Foram utilizados artigos científicos e ainda documentos oficiais como base teórica.

Para a realização da revisão bibliográfica desse estudo foram utilizadas as seguintes bases de dados: Biblioteca Virtual de Saúde (BVS) e PubMED. Foram utilizados como descritores: climatério, menopausa e saúde da mulher.

Foram incluídos artigos científicos no idioma inglês e português, publicados nos anos de 2010 a 2020 e disponíveis na íntegra por via eletrônica (Internet) e que se aproximassem da temática estudada. Excluídos textos não originais, que apresentavam fuga ao tema ou duplicidade nas bases de dados, podemos observa no fluxograma 1, as estratégias de busca utilizadas nas bases de dados.

Fluxograma 1: Estratégia de busca nas bases de dados.

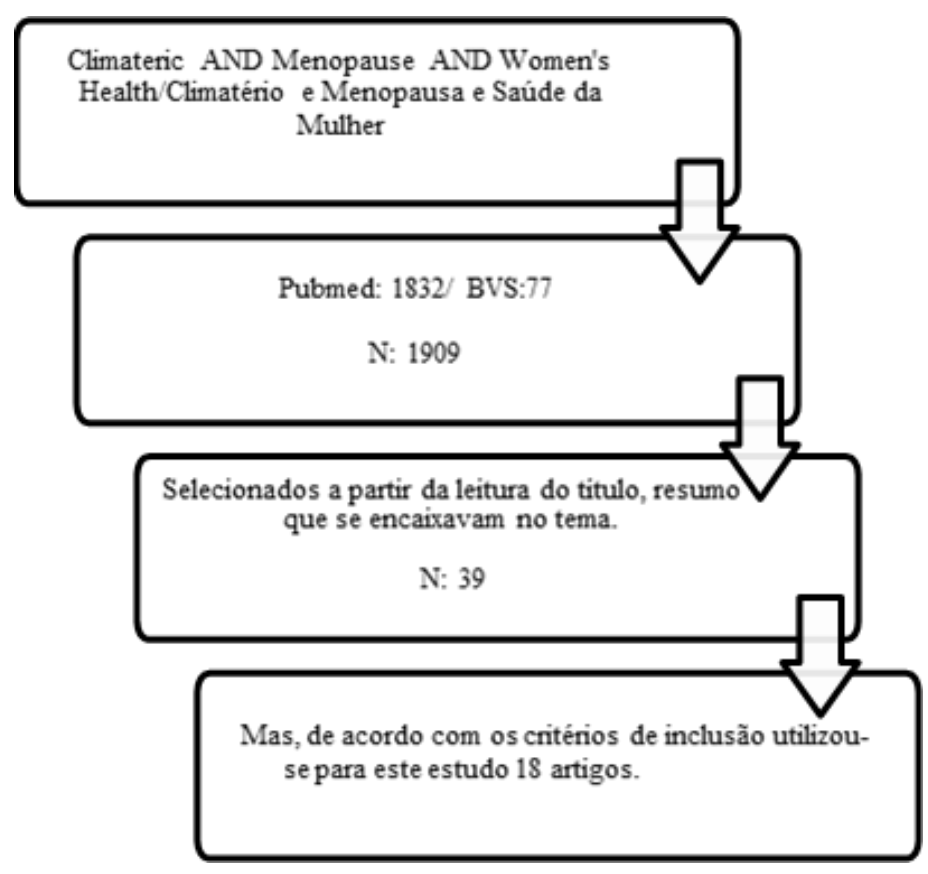

Fonte: Autores (2020). 
Feito a seleção dos artigos encontrados, ordenamento do material e classificação por semelhança semântica, agrupadas de acordo com a semelhança de conteúdo, sendo distribuídas em três categorias.

Este estudo foi realizado nas seguintes categorias temáticas: Impacto do Climatério no Aspecto Físico e na Vida Social; A sexualidade durante o Climatério e Assistência de Enfermagem na Fase do Climatério.

\section{Resultados}

Para realização desta pesquisa bibliográfica foram revisadas onze (11) artigos científicos e sete (07) revisões bibliográficas, com diferentes abordagens, mas que estão de acordo com o tema proposto. Abaixo no Quadro 2 serão descritos os estudos realizados para chegar aos resultados e conseguintemente estabelecer discussões.

Quadro 2: Revisão dos artigos inclusos na revisão bibliográfica (título do artigo, ano e nome dos autores).

\begin{tabular}{|c|c|c|}
\hline TÍTULO & ANO & AUTOR \\
\hline $\begin{array}{l}\text { Mulher no Climatério: reflexões sobre desejo } \\
\text { sexual, beleza e feminilidade,São Paulo. }\end{array}$ & 2010 & $\begin{array}{l}\text { Valença, C. N., do Nascimento Filho, J. M., \& } \\
\text { Germano, R. M. }\end{array}$ \\
\hline Percepções de mulheres acerca doclimatério. & 2018 & Piecha, V. H., et al. \\
\hline $\begin{array}{c}\text { Mudanças comportamentais e fisiológicas } \\
\text { determinadas pelo ciclo biológico feminino - } \\
\text { climatério àmenopausa. }\end{array}$ & 2018 & Selbac, M. T., et al. \\
\hline $\begin{array}{c}\text { Menopausa, Sinais e Sintomas e seus Aspectos } \\
\text { Psicológicos em Mulheres sem Uso de } \\
\text { Reposição Hormonal. }\end{array}$ & 2015 & Ferreira, I. C. C., Silva, S. S., \& de Almeida, R. S. \\
\hline $\begin{array}{l}\text { A influência dos sintomas climatéricosna } \\
\text { saúde da mulher. }\end{array}$ & 2020 & Figueiredo Júnior, J. C., et al. \\
\hline $\begin{array}{llll}\text { A } & \text { percepção } & \text { do } & \text { climatério }\end{array}$ & 2019 & Serpa, M. A. \\
\hline $\begin{array}{l}\text { Alterações biopsicossociais na mulher } \\
\text { climatérica: uma revisão bibliográfica. }\end{array}$ & 2013 & $\begin{array}{c}\text { Veloso, L. C., Maranhão, R. M. S., \& Lopes, V. M. } \\
\text { L.V. }\end{array}$ \\
\hline $\begin{array}{c}\text { Sintomas psicológicos em mulheres } \\
\text { climatéricas cardiopatas. }\end{array}$ & 2018 & Nogueira, J. S. et al. \\
\hline $\begin{array}{c}\text { Symptoms and diseases associatedwith } \\
\text { climacteric and menopause. }\end{array}$ & 2019 & Sarduy, M. I. T. et al. \\
\hline $\begin{array}{l}\text { Vida da Mulher no Climatério: Um } \\
\text { mapeamento das alterações manifestadas. }\end{array}$ & 2011 & De Bitencourt, C. C. et al. \\
\hline $\begin{array}{c}\text { Vivenciando o climatério: percepções e } \\
\text { vivencias de mulheres atendidas na atenção } \\
\text { básica. }\end{array}$ & 2018 & Vieira, M. M. et al. \\
\hline $\begin{array}{l}\text { Influências do climatério emrelacionamentos } \\
\text { conjugais: perspectivade gênero. }\end{array}$ & 2018 & Carvalho, M. L de. et al. \\
\hline $\begin{array}{c}\text { Violência por parceiro intimo em abuso de } \\
\text { álcool perpetrada contra mulheres no } \\
\text { climatério. }\end{array}$ & 2019 & Pires, V. M. M. M .et al. \\
\hline O período climatério sob ótica da mulher. & 2019 & Da Silva, M. G., Dias, M. S., \& Oliveira, M. P. \\
\hline $\begin{array}{l}\text { Atuação de enfermeiros na atenção àsmulheres } \\
\text { no climatério. }\end{array}$ & 2015 & Da Silva, C. B. et al. \\
\hline $\begin{array}{c}\text { Assistência de Enfermagem à Mulheres no } \\
\text { Climatério. }\end{array}$ & 2020 & Silva, A. P. A. A., \& Pontes, L. de Souza. \\
\hline $\begin{array}{l}\text { O conhecimento produzido acerca de } \\
\text { climatério. }\end{array}$ & 2018 & Soares, G. R. de Souza. et al. \\
\hline $\begin{array}{c}\text { Fatores associados à qualidade devida em } \\
\text { mulheres no climatério. }\end{array}$ & 2016 & Serpa, M. A. et al. \\
\hline
\end{tabular}

Fonte: Autores (2020). 


\section{Discussão}

\subsection{Impacto do Climatério no Aspecto Físico e na Vida Social da Mulher}

$\mathrm{O}$ enfrentamento dos processos ligados ao climatério e à menopausa vai além das mudanças hormonais que ocorrem durante este estágio; é aqui que existe uma maior prevalência de processos crônicos, cujos sintomas não estão necessariamente associados à diminuição da produção de estrogênio. Existem vários processos, em torno da menopausa, passam por diversos eventos pessoais, familiares e de trabalho, que podem influenciar a qualidade de vida das mulheres (Salinas, 2017 apud Sarduy, et al., 2019).

O climatério é visto de maneira negativa pela maioria das mulheres que estão vivenciando este período, as mulheres não estão preparadas para enfrentar este período; e as mudanças corporais e estéticas são percebidas de uma forma negativa (Piecha et al., 2018).

A concepção de envelhecimento na sociedade brasileira colabora para uma visão negativa dessa fase, associa-se o envelhecimento com a perda das funções sociais e físicas (Serpa, et al., 2016).

Já De Bitencourt et al. (2011) em seu estudo observou que não houve estas "preocupações" com a velhice, nem com a perda da feminilidade, pois nem sempre isso ocorre em virtude do climatério. Mas o que demostra sentimentos de preocupação com alterações desagradáveis, dentre eles a secura vaginal, dispareunia e incontinência urinária.

Algumas mulheres no seu ver e da sociedade elas perdem a sua funcionalidade, assim diminuindo sua possibilidade de felicidade, pois durante este período perdem os atributos conquistados com a puberdade, como a beleza, o vigor, fertilidade; portanto, colocando sua autoestima, exclusivamente, na sua capacidade de conceber e na beleza de seu corpo, se sentindo desvalorizada e, sem encontrar algum sentido em sua vida (Mendonça, 2004 apud Veloso, Maranhão \& Lopes, 2013).

O preconceito social e o desconhecimento pela mulher sobre as mudanças ocorridas nesse período se constituem em barreiras, afetando os cuidados necessários com a saúde e, consequentemente, prejudicando sua qualidade de vida (Cardoso, De Camargo apud Nogueira et al., 2018.).

Tais fatores são relativos e cada mulher vivencia de forma diferente, algumas mais e outras menos prejuízo em suas atividades diárias. Dar-se a relevância da orientação a respeito destas mudanças comportamentais, psíquicas e principalmente fisiológicas que ocorrem na ancianidade (Oliveira Júnior, 2016).

Ferreira, Silva \& de Almeida (2015, p.63) relatam em seu estudo "que de maneira geral a falta de informação está diretamente relacionada à baixa renda familiar, baixo nível de escolaridade, frequência irregular nas consultas ginecológicas e a idade e que estes fatores são associados a não compreensão da menopausa, seus sinais e sintomas e tratamento".

Os mesmos autores detectam em seu estudo que os principais fatores como alterações como os fogachos, a depressão e a redução da libido ocasionam em algumas, diminuição do desejo sexual, de tal maneira que desestrutura sua família e algumas levando ao fim do casamento, além de afetar seu convívio social e desempenho no trabalho.

Estas alterações é relatado por Figueiredo Júnior et al. (2020, p. 4001) que os sintomas climatéricos, os fogachos, somáticos, levam ao estresse levando as mulheres a se tornarem queixosas e muitas vezes tratadas com indiferença nos serviços de saúde.

\subsection{A Sexualidade Durante o Climatério}

O envelhecimento atinge a mulher e o homem, pois, os valores sócios, culturais, econômicos e políticos, delegam à função reprodutora a mulher e o homem exerce uma função dominante na relação, este aspecto atinge a sexualidade. Muitas das vezes a relação entre ambos os sexos não se trata do desejo, do amor, da paixão, mas sim pela imposição das práticas políticas, econômicas e culturais que necessitam de um parceiro e filhos para serem bem sucedidos dentro da sociedade 
(Pinotti; Halbe \& Hegg, 1995 apud Valença, Nascimento Filho \& Germano, 2010).

Para a sociedade quando a mulher inicia o climatério no seu processo de envelhecimento, e da ressignificação de sua sexualidade num período pós- reprodutivo, consequentemente ela sofre a perda do desejo sexual, mas é nada além de um mito agregando a mulher como objeto da procriação e da incapacidade de transcender a metamorfose física para uma nova esfera psíquica e social (Valença, Nascimento Filho \& Germano, 2010).

Em um estudo feito por Selbac et al., (2018) obteve dados divergentes do autor acima; que durante o climatério uma das principais queixas é a inapetência sexual, o hipoestrogenismo atinge bruscamente as características fenotípicas da mulher, a beleza feminina, fatores relevantes no meio social e cultural na atração pelo sexo oposto e na sua autoimagem. A fisiologia e sexualidade feminina é influenciada através do estrogênio, que age nos orgãos sexuais. A disfunção sexual causa vulnerabilidade na vida sexual da mulher no climatério, provocados por fatores físicos, sociais e psicológicos e as alterações hormonais que provocam diversos efeitos nos órgãos genitais e no sistema nervoso (Ferreira, Silva \& Almeida, 2015 apud Vieira, 2018).

O climatério faz com que a mulher tenha diminuição ou ausência da libido e, consequente, reduzindo a frequência da atividade sexual, afetando diretamente na vivência do relacionamento conjugal. Também, é evidente que a relação sexual ocorre de forma passiva, apenas para que ocorra a satisfação do cônjuge. A dispareunia (dor durante a relação) é outro fator que interfere na relação sexual (Carvalho et al., 2018).

Em consequências das mudanças físicas as interações sexuais tornam-se difíceis e, por isso, evitam ter vida sexual ativa, utilizando o desconforto como principal pressuposto (Soares et al., 2018).

Estudos demostraram que a prevalência de disfunção sexual nas mulheres entre 40 e 65 anos é de $67 \%$, tendo $60 \%$ das mulheres afirmando que a atividade sexual diminui após a menopausa (SANTOS et al., 2014 apud PIRES et al, 2019).

Serpa et al. (2016) demostra em sua pesquisa que as participantes perceberam um desejo sexual diminuído e relataram como responsáveis por essa diminuição da libido as mudanças no corpo e os sintomas associados ao climatério. Demostrando também um receio por parte da visão do companheiro em relação à incompreensão deles acerca desse momento e deixaram transparecer certo medo de término do relacionamento.

Carvalho et al. (2018), observaram em seu estudo influências positivas do climatério em relacionamentos conjugais, sob a ótica das mulheres participantes, houvendo intensificação, por parte do cônjuge, de atitudes de carinho, companheirismo, compreensão, respeito e diálogo.

Para melhor vivencia da sexualidade durante o climatério a Estratégia Saúde da Família (ESF), é a principal entrada para estas mulheres que necessitam de informações e auxilio nesta fase da vida (Rocha; Rocha, 2010 apud Da Silva, Dias \& Oliveira, 2019).

As mulheres no período do climatério, assim como na menopausa, vivem um momento delicado de transformações físicas, papel social, e na forma como essas mulheres se posicionam frente a preconceitos e tabus, o que pode interferir na maneira como sua sexualidade é vivênciada, e no modo como buscam a felicidade (Serpa, 2019).

\subsection{Assistência de Enfermagem na Fase do Climatério}

A saúde da mulher é um campo de atuação bastante complexo, que exige total dedicação em todas as suas ações, com atenção integral às mulheres e não apenas necessariamente em algumas estratégias específicas (Silva et al., 2015). A enfermagem como atua durante a consulta de enfermagem por meio da compreensão do climatério como uma etapa que envolve questões biopsicossociais (Piecha et al., 2018).

No período climatério uma assistência à saúde da mulher com profissionais que tenham conhecimento sobre as manifestações clinica e alterações fisiológicas que abrange o climatério são primordiais para oferecer um serviço de qualidade 
a fim de proporcioná-las uma qualidade de vida com maior segurança e bem-estar neste período (Bitencourt et al., 2011).

O mesmo autor retrata a enfermagem como base na atividade do cuidar, onde sua especificidade é o cuidar do ser humano. Que abrange o paciente, a família e a comunidade, buscando compreender e promover o cuidado, colocando em prática atividades de promoção e prevenção da saúde. É essencial no climatério, pois a mulher necessita de cuidado e informação acerca do que está vivenciando ou que irá vivenciar neste período.

Silva e Pontes (2020, p. 14) ressalva a importância do profissional de enfermagem adotar uma ampla abordagem biopsicossocial, onde se foca na melhoria da qualidade de vida e nas necessidades de cada mulher, vista como indivíduos ativos no êxito e manutenção de um estado de saúde propício ao longo de sua vida.

Acredita-se que, desta forma, as mulheres realmente terão suas necessidades supridas de forma eficiente, em todas as fases de sua vida e, em diferentes aspectos, como recomendam as Políticas Públicas de Saúde brasileiras (Silva et al., 2015).

Silva, et al. (2015, p. 317) afirma que existe a necessidade de incentivo e capacitação dos profissionais da enfermagem para a realização de ações referentes ao climatério, que possam ser abordados por meio de estratégias de educação permanente na UBS.

\section{Conclusão}

O climatério é visto como fator biológico na vida da mulher, o qual é um período que requer bastante atenção, pois lidar com o desconhecido é assustador principalmente quando isto envolve algo sobre o corpo, assim é a mulher no auge da vida passando por uma fase nada fácil, que causa mudanças físicas, psicossociais, sexuais, profissional e conjugal de acordo com os autores estudados.

Foi evidenciado que cada mulher vivencia o climatério de forma singular, tanto como a idade em que se inicia, a forma que acontece se é de forma natural ou cirúrgica; a sintomatologia tem um impacto diferente para cada uma, pois a intensidade é variável.

O modo em que a sociedade ver esta mulher tem bastante interferência no seu psicológico, por ser vista somente como uma reprodutora e a partir do momento em que se torna infértil a sua funcionalidade acaba, e deixando com o psicológico abalado.

A família e o companheiro são vistos como peças essências nesta fase, dando apoio emocional para a mulher, de certo modo sendo compreensivo a ponto de entender que esta etapa da vida é complexa, lhe modificando de vários modos.

Também foi evidenciada a grande importância na busca de conhecimento pelo enfermeiro sobre o assunto, pois, é ele que lida diretamente com a mulher em todos os aspectos, do inicio de sua vida, na puberdade, na gestação e no fim do clico da fertilidade.

Com este conhecimento o enfermeiro é capaz de proporcionar um assistência correta a estas mulheres que lhe procuram, fazendo com que a mesma se sinta bem assistida por um profissional capacitado, tirando suas dúvidas geradas neste ciclo de sua vida.

No que tange às contribuições do presente estudo, acredita-se que poderá facilitar no desenvolvimento de promoção, prevenção, como também na reabilitação de saúde. Já quanto ao ensino e pesquisa, espera-se que as argumentações e reflexões proporcionadas pelos dados encontrados, forneçam instrumentos necessários para formação em enfermagem, assim como nas demais aréas da saúde, contribuindo para a qualificação do ensino na saúde da mulher, principalmente no que concerne o contexto do climatério. 


\section{Referências}

Brasil. (2008). Ministério da Saúde. Manual de Atenção à Mulher no Climatério/ Menopausa. http://bvsms.saude.gov.br/bvs/publicacoes/cade rno_atencao_basica_diretrizes_nas f.pdf.

Brasil. (2016). Ministério da Saúde. Protocolo de Atenção Básica Saúde das Mulheres. http//bvsms.saude.gov.br/bvs/publicacoes/prot ocolos_atencao_basica_saude_m ulheres.pdf.

Da Silva, C. B. et al. (2015). Atuação de Enfermeiros na Atenção às Mulheres no Climatério. Rev. Enferm. UFPE on line, 312-318. https://periodicos.ufpe.br/revistas/revistaenfermagem/article/view/10341/11047.

Da Silva, M. G., Dias, M. S.; \& Oliveira, M. P. (2019). O período climatérico sob ótica da mulher. Saber Digital. 12(1), 29-38. http://revistas.faa.edu.br/index.php/SaberDigital/article/view/723.

De Bitencourt, C.C. et al. (2011). Vida da Mulher no Climatério: Um mapeamento das alterações manifestadas. Revista Eletrônica de Comunicação, Informação e Inovação em Saúde, 5(3). https://homologacao-reciis.icict.fiocruz.br/index.php/reciis/article/view/565.

De Carvalho, M. L. et al. (2018). Influências do climatério em relacionamentos conjugais: perspectiva de gênero. http://repositorio.ufc.br/handle/riufc/37645.

Ferreira, I. C. C., Silva, S. S., \& de Almeida, R. S. (2015). Menopausa, sinais e sintomas e seus aspectos psicológicos em mulheres sem uso de reposição hormonal. Ensaios e Ciência: C. Biológicas, Agrárias e da Saúde. 19(2). https://revista.pgsskroton.com/index.php/ensaioeciencia/article/view/3182.

Figueiredo Júnior, J. C., et al. (2020). A influência dos sintomas climatéricos na saúde da mulher. Nursing (São Paulo), 3996-4001. https://pesquisa.bvsalud.org/portal/resource/pt/biblio-1102701?src=similardocs.

Febrasgo, Comissões Nacionais Especializadas Ginecologia e Obstetrícia. (2010). Climatério, Manual de Orientação em Climatério.

Nogueira, J. S. et al. (2018). Sintomas psicológicos em mulheres climatéricas cardiopatas. Cogitare Enfermagem, 23(2).

Oliveira Júnior, M. L. de. (2016). Climatério-principais alterações fisiológicas, emocionais e sociais que ocorrem nas mulheres.

Piecha, V. H. et al. Women's insights about the climacteric period/Percepções de mulheres acerca do climatério. Revista de Pesquisa: Cuidado é Fundamental Online, 10(4), 906-912. http://www.seer.unirio.br/index.php/cuidadofundamental/article/download/6259/pdf.

Pires, V. M. M. M. et al. (2019). Violência por parceiro intimo em abuso de álcool perpetrada contra mulheres no climatério. Revista de Enfermagem da UFSM, 9, 45. https://periodicos.ufsm.br/reufsm/article/view/34201/html.

Santos, L. M. et al. (2007). Síndrome do climatério e qualidade de vida: uma percepção das mulheres nessa fase da vida. Revista APS, 10(1), 20-26. http://www.ufjf.br/nates/files/2009/12/Climaterio.pdf.

Sarduy, M. I. T. et al. (2019). Symptoms and diseases associated with climacteric and menopause. Medicentro,23(2), 116-124. https://www.medigraphic.com/cgi- bin/new/resumenI.cgi?IDREVISTA=298\&IDARTICULO=86749\&IDPUBLICACION=8304.

Selbac, M. T., et al. (2018). Mudanças comportamentais e fisiológicas determinadas pelo ciclo biológico feminino-climatério à menopausa. Aletheia, 51(1,2). http://www.periodicos.ulbra.br/index.php/aletheia/article/view/4921.

Serpa, M. A. et al. (2016). Fatores associados à qualidade de vida em mulheres no climatério. Reprodução \& Climatério, 31(2), 76-81. https://www.sciencedirect.com/science/article/pii/S1413208716300152.

Silva, A. P. A. A., \& Pontes, L. S. (2020). Assistência de Enfermagem à Mulheres No Climatério. https://dspace.uniceplac.edu.br/handle/123456789/355.

Soares, G. R. de Souza. et al. (2018). O conhecimento produzido acerca de climatério, família e envelhecimento. Rev. enferm. UERJ, e32588-e32588. https://www.e- publicacoes.uerj.br/index.php/enfermagemuerj/article/view/32588.

Una-Sus, UFMA. (2013). Curso de Especialização, Saúde da mulher geral, São Luís, MA. https://ares.unasus.gov.br/acervo/.../Provab2012.1_Modulo11_Introducao.pdf..

Valença, C. N., do Nascimento Filho, J. M., \& Germano, R. M. (2010). Mulher no climatério: reflexões sobre desejo sexual, beleza e feminilidade. Saúde e Sociedade, 19, 273-285. https://www.scielosp.org/article/sausoc/2010.v19n2/273-285/pt/.

Veloso, L. C., Maranhão, R. M. S., Lopes, V. M. L.V. (2013). Alterações biopsicossociais na mulher climatérica: uma revisão bibliográfica. Revista Interdisciplinar, 6(3), 187-194.

Vianna, I. O. (2010). Metodologia do trabalho científico: um enfoque didático da produção científica. EPU.

Vieira, T. M. M. et al. (2018). Vivenciando o climatério: percepções e vivencias de mulheres atendidas na atenção básica. Enfermagem em foco, 9(2). http://revista.cofen.gov.br/index.php/enfermagem/article/view/1084. 